\title{
ASSESSMENT OF BRUCELLOSIS KNOWLEDGE, ATTITUDE AND PRACTICE AMONG VETERINARIANS IN INDIA
}

\section{Govindaraj $\mathrm{G}^{1, *}$, Nagalingam $\mathrm{M}^{1}$, Nethrayini $\mathrm{KR}^{1}$, Shalini $\mathrm{R}^{1}$, Rajeswari Shome ${ }^{1}$, Bambal $\mathrm{RG}^{2}$, Lipi Sairiwal ${ }^{2}$ and Rahman $\mathrm{H}^{3}$}

${ }^{1}$ Indian Council of Agricultural Research-National Institute of Veterinary Epidemiology and Disease Informatics (ICAR-NIVEDI), Ramagondanahalli, Yelahanka, Bengaluru, India-560 064.

${ }^{2}$ Ministry of Agriculture and Farmers Welfare, Department of Animal Husbandry, Dairying \& Fisheries, Krishi Bhavan, New Delhi-110 001.

${ }^{3}$ Animal Science Division, ICAR, Krishi Bhavan, New Delhi-110 001.

Received - August 2, 2016; Revision - October 15, 2016; Accepted - October 27, 2016

Available Online - October 30, 2016

DOI: http://dx.doi.org/10.18006/2016.4(Spl-3-ADPCIAD).S83.S94

\section{KEYWORDS \\ Brucellosis \\ Knowledge Index \\ Attitude Index \\ Practice Index \\ Brucellosis Control \\ Programme}

\begin{abstract}
Brucellosis is considered as one of the major zoonotic infections worldwide. However, there is paucity of studies on knowledge, attitude and practice level of high risk groups like veterinarians in India. This study attempted to address this gap by conducting cross-sectional survey in four states of India (Assam, West Bengal, Uttar Pradesh and Punjab states). The data was collected from 160 veterinarians through the self-administered pre-tested questionnaire. There exist wide differences in knowledge level on brucellosis among veterinarians across the surveyed states. In all the studied states less than $12 \%$ of the veterinarians were fully aware on the nuances of Brucellosis Control Programme implemented in India indicating the need for appropriate intervention to achieve the intended targets. The knowledge, attitude and practice Index of majority of the veterinarians ranged between 74 to 85,58 to 71 and 86 to 100 , respectively. The knowledge on brucellosis is significantly correlated with the prevalence in the states (except West Bengal) indicating veterinarians in higher brucellosis prevalence states have better knowledge. There exists significant difference across the states in knowledge and attitude level on brucellosis indicating the need of imparting appropriate training to upgrade knowledge and to build positive attitude among veterinarians.
\end{abstract}

* Corresponding author

E-mail: mggraj74@gmail.com (Govindaraj G)

Peer review under responsibility of Journal of Experimental Biology and Agricultural Sciences.

Production and Hosting by Horizon Publisher India [HPI] (http://www.horizonpublisherindia.in/).

All rights reserved
All the article published by Journal of Experimental Biology and Agricultural Sciences is licensed under a Creative Commons Attribution-NonCommercial 4.0 International License Based on a work at www.jebas.org.

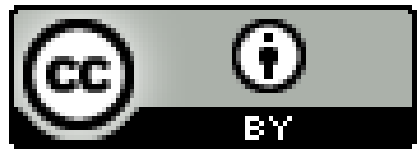




\section{Introduction}

Brucellosis is regarded as one of the major zoonotic infections worldwide. More than 500000 new cases occur annually but with an uneven global distribution (Skalsky et al., 2008). It remains an uncontrolled problem in regions of high endemicity such as Mediterranean, Middle East, Africa, Latin America and parts of Asia (Refai, 2002). Species infecting domestic animals are Brucella melitensis (goats and sheep), B. suis (pigs), $B$. abortus (cattle and bison), B. ovis (sheep), and B. canis (dogs) (Gull \& Khan, 2007).

In India, bovine brucellosis is widespread and appears to be on the increase in recent times, perhaps due to increased trade and rapid movement of livestock (Renukaradhya et al., 2002). The increase in bovine brucellosis increases the risk of spread of the disease to veterinary professionals, especially, the field veterinarians who treats the animals on the daily basis. Brucellosis prevalence varied widely across the livestock and human population in different states of India. The seroprevalence of brucellosis in milch goats and humans was $11.45 \%$ and $2.97 \%$, respectively (Kapoor et al., 1985). In Gujarat state, $8.5 \%$ prevalence of Brucella agglutinins was recorded in human cases (Panjarathinam \& Jhala, 1986). The various levels of brucellosis sero-prevalence across the species and states in India are reported by researchers (Rajkhowa et al., 2005; Londhe et al., 2011; Ramesh et al., 2013).

Brucellosis causes considerable economic loss in livestock and humans. In livestock, brucellosis causes reduced productivity, abortion, and weak offspring. The death of animal may also occur as a result of acute metritis, followed by retained fetal membranes (Radostitis et al., 2000). In humans, bone and joint involvement is the most frequent complication of brucellosis (Mousa et al., 1987). Besides affecting the healthy life in humans, brucellosis causes considerable financial burden of the family like increased cost of hospital treatment, cost of drugs, patient out-of pocket treatment expenses, loss of work or income loss due to illness, and Disability Adjusted Life Year (DALY) burden. During pregnancy brucellosis poses a substantial risk of spontaneous abortion or intrauterine transmission of infection to the infant (Hajia et al., 2009). The veterinary professionals who treat animals on daily basis form the high risk group for brucellosis. If the set practices are not followed by the veterinarians especially for the brucellosis suspected cases, might result in infection of this deadly disease to the veterinarians. In the long-run it aids the spread to their partners (Shome et al., 2014). Thus, brucellosis prevention, control and eradication are very important for any country to protect humans and animals. Considering the economic losses in livestock and zoonotic importance of the disease, Government of India implemented Brucellosis Control Programme (Brucellosis CP) during 2010 for mass screening followed by vaccination in areas where incidence of disease is high. The aim of Brucellosis-CP is to reduce economic loss and to reduce impact of the disease on human health besides mitigating the loss due to infection in animals. The success of public health programme like Brucellosis CP implemented in India depends on the Knowledge, Attitude and Practice (KAP) of the field level implementing officials like veterinarians. There is paucity of studies on KAP and hence this study attempted to address this gap by conducting cross-sectional survey among veterinarians in important states of Northern India.

\section{Materials and Methods}

\subsection{Study area}

The study was performed in four states of India (Assam, West Bengal, Uttar Pradesh and Punjab) during 2013-14. Assam is in north-eastern region with a livestock population of 19 million; West Bengal is in eastern part of India with livestock population of 30 million; Uttar Pradesh is located in northern India with livestock population of 68 million; and Punjab is in the north-western region of India with livestock population of 8 million (DAHD \& F, 2015).

\subsection{Sample size}

A two stage proportionate sampling technique was adopted for the primary survey to assess the KAP levels of veterinary professionals. In the first stage, four states in India viz., Assam, West Bengal, Uttar Pradesh and Punjab were selected randomly. In the second stage, the proportionate sampling technique was adopted to select the number of respondents from each of the selected states based on the number of veterinarians working in the state. The selection of the sample size for this study was based on table developed by Bartlett et al., 2001. The sample size for a population 4,000 to 6,000 ranges from 198 to 209 for 0.03 margin error and 0.01 alpha. Accordingly, based on number of veterinarians working in the states (5123), the samples targeted for survey were 200. Out of 200 samples targeted, 160 participated and filled the knowledge component of the questionnaire, whereas, only 148 and 132 veterinarians participated in the attitude and practice component of the questionnaire, respectively. For operational feasibility, the survey was conducted during one day orientation programme on Brucellosis CP organized by the Animal Husbandry Department of states along with authors institute with the support of DAHD\&F, India. Hence, for the present study the data was collected from the veterinarians who attended this orientation.

\subsection{Questionnaire Development and Administration}

The authors institute organizes various epidemiological trainings on brucellosis for the benefit of field veterinarians in India. During these trainings the KAP questionnaires developed by the authors were pre-tested and modified accordingly. Thus, a standard, pre-tested, structured questionnaire developed was self-administered to veterinarians working in different states of India during the main survey. 
Table 1 Description and format of questions used to collect information on KAPs from the respondents.

Topic

Knowledge statements

A. Brucella organism

About the Brucella organism Details

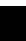

Participants have to ascertain the type of organism, mode of invasion, excretion and complexity of the organism.

\begin{tabular}{|c|c|c|}
\hline Clinical signs of brucellosis & $\begin{array}{l}\text { Participants have to ascertain the disease symptoms in male and female animals like occurrence of abortion in any point of } \\
\text { pregnancy, abortion occurs mostly during third trimester with leathery placenta and symptoms in male animals are epididymitis, } \\
\text { orchitis and hygroma. }\end{array}$ & Closed ended (Yes /No) \\
\hline Source of infection in livestock & $\begin{array}{l}\text { Participants have to identify the possible source of infection like aborted fetuses, afterbirth and vaginal discharge. Questions were } \\
\text { separately formulated to investigate other source of infection like pastures, fodder and water. Questions on dam to calf infection and } \\
\text { using the semen straw produced from infected bulls were also provided. }\end{array}$ & Closed ended (Yes /No) \\
\hline Causes of brucellosis in humans & $\begin{array}{l}\text { Participants have to identify the sources of infection like physical contact, assisting parturition, contact with fetal membrane, } \\
\text { drinking raw milk etc. }\end{array}$ & Closed ended (Yes /No) \\
\hline Vaccination against brucellosis & $\begin{array}{l}\text { Participants have to identify the type of vaccine against brucella, about age and sex of the animals to be vaccinated, any booster } \\
\text { dose requirement, and immunity level of the vaccine in the animals etc. }\end{array}$ & Closed ended (Yes /No) \\
\hline $\begin{array}{l}\text { Brucellosis Control Programme } \\
\text { (Brucellosis CP) }\end{array}$ & Participants have to identity the correct statements related to Brucellosis CP. & Closed ended (Yes /No) \\
\hline Others aspects of brucellosis & $\begin{array}{l}\text { Questions were also developed to assess the knowledge level on the susceptibility of breeds, age groups and hazard nature for the } \\
\text { professionals. }\end{array}$ & Closed ended (Yes /No) \\
\hline Attitude statements & $\begin{array}{l}\text { Attitude towards the importance of brucellosis, its spread, control programme, disease tagging to monitor disease and farmers } \\
\text { participation in the control programme }\end{array}$ & $\begin{array}{l}\text { Likert scale (agree/ neutral/ } \\
\text { disagree) }\end{array}$ \\
\hline Practice statements & $\begin{array}{l}\text { Questions were developed to assess the practice of usage of protective gears by veterinarians, sending suspected samples to labs, } \\
\text { advisory service to farmers to control brucellosis at farm level }\end{array}$ & Closed ended (Yes /No) \\
\hline
\end{tabular}

Table 2 Knowledge on different aspects of brucellosis and Brucellosis CP in the study states.

\begin{tabular}{|c|c|c|c|c|c|c|c|c|c|c|}
\hline & \multicolumn{2}{|l|}{ Assam } & \multicolumn{2}{|l|}{ Punjab } & \multicolumn{2}{|c|}{ Uttar Pradesh } & \multicolumn{2}{|l|}{ West Bengal } & \multicolumn{2}{|l|}{ Pooled } \\
\hline Knowledge statements & No. of Respondents & $\%$ & No. of Respondents & $\%$ & No. of Respondents & $\%$ & No. of Respondents & $\%$ & No. of Respondents & $\%$ \\
\hline \multicolumn{11}{|c|}{ About Brucella organism } \\
\hline $4 / 4$ & 15 & 60 & 8 & 25 & 17 & 27 & 11 & 28 & 51 & 32 \\
\hline $3 / 4$ & 7 & 28 & 19 & 59 & 30 & 48 & 23 & 57 & 79 & 49 \\
\hline $2 / 4$ & 3 & 12 & 3 & 10 & 12 & 19 & 6 & 15 & 24 & 15 \\
\hline \multicolumn{11}{|c|}{ Clinical signs on brucellosis } \\
\hline $3 / 3$ & 15 & 60 & 27 & 84 & 48 & 76 & 32 & 80 & 122 & 76 \\
\hline $2 / 3$ & 8 & 32 & 4 & 13 & 15 & 24 & 7 & 18 & 34 & 21 \\
\hline \multicolumn{11}{|c|}{ Brucella Infection in livestock } \\
\hline $4 / 4$ & 6 & 24 & 24 & 75 & 36 & 57 & 30 & 75 & 96 & 60 \\
\hline
\end{tabular}

Journal of Experimental Biology and Agricultural Sciences

http://www.jebas.org 


\begin{tabular}{|c|c|c|c|c|c|c|c|c|c|c|}
\hline $3 / 4$ & 13 & 52 & 6 & 19 & 21 & 33 & 4 & 10 & 44 & 28 \\
\hline $2 / 4$ & 6 & 24 & 2 & 6 & 6 & 10 & 4 & 10 & 18 & 11 \\
\hline \multicolumn{11}{|c|}{ Causes/chances(zoonotic potential) of brucellosis to humans } \\
\hline 7/7 & 5 & 20 & 4 & 13 & 7 & 11 & 6 & 15 & 5 & 20 \\
\hline $6 / 7$ & 8 & 32 & 22 & 69 & 35 & 56 & 18 & 45 & 8 & 32 \\
\hline $5 / 7$ & 7 & 28 & 5 & 16 & 10 & 16 & 6 & 15 & 7 & 28 \\
\hline 4/7 & 2 & 8 & 1 & 3 & 7 & 11 & 6 & 15 & 2 & 8 \\
\hline $3 / 7$ & 2 & 8 & 0 & 0 & 1 & 2 & 2 & 5 & 2 & 8 \\
\hline $2 / 7$ & 0 & 0 & 0 & 0 & 1 & 2 & 1 & 3 & 0 & 0 \\
\hline \multicolumn{11}{|c|}{ Vaccination against brucellosis } \\
\hline $6 / 6$ & 2 & 8 & 21 & 66 & 17 & 27 & 27 & 68 & 67 & 42 \\
\hline $5 / 6$ & 17 & 68 & 9 & 28 & 23 & 37 & 11 & 28 & 60 & 38 \\
\hline $4 / 6$ & 5 & 20 & 1 & 3 & 12 & 19 & 0 & 0 & 18 & 11 \\
\hline $3 / 6$ & 1 & 4 & 1 & 3 & 8 & 13 & 0 & 0 & 10 & 6 \\
\hline $2 / 6$ & 0 & 0 & 0 & 0 & 2 & 3 & 1 & 3 & 3 & 2 \\
\hline \multicolumn{11}{|c|}{ Brucellosis Control Programme (Brucellosis-CP) } \\
\hline $4 / 4$ & 3 & 12 & 3 & 9 & 5 & 8 & 5 & 13 & 16 & 10 \\
\hline $3 / 4$ & 4 & 16 & 3 & 9 & 9 & 14 & 13 & 33 & 29 & 18 \\
\hline $2 / 4$ & 8 & 32 & 13 & 41 & 15 & 24 & 12 & 30 & 48 & 30 \\
\hline \multicolumn{11}{|c|}{ Other aspects of brucellosis } \\
\hline $3 / 3$ & 1 & 4 & 7 & 22 & 43 & 68 & 22 & 55 & 73 & 46 \\
\hline $2 / 3$ & 12 & 48 & 24 & 75 & 16 & 25 & 14 & 35 & 66 & 41 \\
\hline
\end{tabular}

Table 3 Knowledge Index on brucellosis in different surveyed states.

\begin{tabular}{|c|c|c|c|c|c|c|c|c|c|c|}
\hline \multirow[t]{2}{*}{ Knowledge Index } & \multicolumn{2}{|c|}{ Assam } & \multicolumn{2}{|c|}{ West Bengal } & \multicolumn{2}{|c|}{ Uttar Pradesh } & \multicolumn{2}{|c|}{$\begin{array}{c}\text { Punjab } \\
\end{array}$} & \multicolumn{2}{|c|}{$\begin{array}{c}\text { Pooled } \\
\end{array}$} \\
\hline & No. of respondents & Cumulative & No. of respondents & Cumulative & No. of respondents & Cumulative & No. of respondents & Cumulative & No. of respondents & Cumulative \\
\hline 19 to 30 & - & 0 & $1(2.5)$ & $1(2.5)$ & $1(1.6)$ & $1(1.6)$ & - & 0 & $2(1.3)$ & $2(1.3)$ \\
\hline 30 to 41 & - & 0 & - & $1(2.5)$ & - & 1(1.6) & - & 0 & - & $2(1.3)$ \\
\hline 41 to 52 & $1(4.0)$ & $1(4.0)$ & $1(2.5)$ & $2(5.0)$ & - & $1(1.6)$ & $1(3.1)$ & $1(3.1)$ & $3(1.9)$ & $5(3.1)$ \\
\hline 52 to 63 & $2(8.0)$ & $3(12.0)$ & $3(7.5)$ & $5(12.5)$ & $3(4.8)$ & $4(6.3)$ & - & $1(3.1)$ & $8(5.0)$ & $13(8.1)$ \\
\hline 63 to 74 & $6(24.0)$ & $9(36.0)$ & $2(5.0)$ & $7(17.5)$ & $16(25.4)$ & $20(31.7)$ & $3(9.4)$ & $4(12.5)$ & $27(16.9)$ & $40(25.0)$ \\
\hline 74 to 85 & $11(44.0)$ & $20(80.0)$ & $14(35.0)$ & $21(52.5)$ & $30(47.6)$ & $50(79.4)$ & $22(68.8)$ & $26(81.3)$ & $77(48.1)$ & $117(73.1)$ \\
\hline 85 to 96 & $5(20.0)$ & $25(100.0)$ & $19(47.5)$ & $40(100.0)$ & $13(20.6)$ & $63(100.0)$ & $6(18.8)$ & $32(100.0)$ & $43(26.9)$ & $160(100.0)$ \\
\hline Total & $25(100.0)$ & & $40(100.0)$ & & $63(100.0)$ & & $46(100.0)$ & & $160(100.0)$ & \\
\hline
\end{tabular}

Figures in parenthesis indicates percentage to the total 
Table 4 Results of attitude levels on brucellosis in different states.

\begin{tabular}{|c|c|c|c|c|c|c|c|c|c|c|c|c|c|c|c|}
\hline \multirow[t]{2}{*}{ Attitude statements } & \multicolumn{3}{|c|}{ Assam } & \multicolumn{3}{|c|}{ West Bengal } & \multicolumn{3}{|c|}{ Uttar Pradesh } & \multicolumn{3}{|c|}{ Punjab } & \multicolumn{3}{|c|}{ Pooled } \\
\hline & A & $\mathrm{N}$ & DA & A & $\mathrm{N}$ & DA & A & $\mathrm{N}$ & DA & A & $\mathrm{N}$ & DA & A & $\mathrm{N}$ & DA \\
\hline $\begin{array}{l}\text { 1. It is a very important disease causing huge econ } \\
\text { other disease in cattle }\end{array}$ & $\begin{array}{c}17 \\
(70.8)\end{array}$ & $\begin{array}{c}6 \\
(25.0)\end{array}$ & $\begin{array}{c}1 \\
(4.2)\end{array}$ & $\begin{array}{c}23 \\
(59.0)\end{array}$ & $\begin{array}{c}2 \\
(5.1)\end{array}$ & $\begin{array}{c}14 \\
(35.9)\end{array}$ & $\begin{array}{c}38 \\
(71.7)\end{array}$ & $\begin{array}{c}5 \\
(9.4)\end{array}$ & $\begin{array}{c}10 \\
(18.9)\end{array}$ & $\begin{array}{c}21 \\
(67.7)\end{array}$ & $\begin{array}{c}5 \\
(16.1)\end{array}$ & $\begin{array}{c}5 \\
(16.1)\end{array}$ & $\begin{array}{c}99 \\
(67.3)\end{array}$ & $\begin{array}{c}18 \\
(12.2)\end{array}$ & $\begin{array}{c}30 \\
(20.4)\end{array}$ \\
\hline 2.It is the top most bacterial disease in outbreak reporting in India & $\begin{array}{c}7 \\
(29.2) \\
\end{array}$ & $\begin{array}{c}11 \\
(45.8) \\
\end{array}$ & $\begin{array}{c}6 \\
(25.0) \\
\end{array}$ & $\begin{array}{c}12 \\
(32.4) \\
\end{array}$ & $\begin{array}{c}2 \\
(5.4) \\
\end{array}$ & $\begin{array}{c}23 \\
(62.2) \\
\end{array}$ & $\begin{array}{c}8 \\
(15.7) \\
\end{array}$ & $\begin{array}{c}17 \\
(33.3) \\
\end{array}$ & $\begin{array}{c}26 \\
(51.0) \\
\end{array}$ & $\begin{array}{c}10 \\
(33.3) \\
\end{array}$ & $\begin{array}{c}10 \\
(33.3) \\
\end{array}$ & $\begin{array}{c}10 \\
(33.3) \\
\end{array}$ & $\begin{array}{c}37 \\
(26.1) \\
\end{array}$ & $\begin{array}{c}40 \\
(28.2) \\
\end{array}$ & $\begin{array}{c}65 \\
(45.8) \\
\end{array}$ \\
\hline $\begin{array}{l}\text { 3. The chance of brucellosis spread in India is fast compared to } \\
\text { western countries }\end{array}$ & $\begin{array}{c}19 \\
(82.6)\end{array}$ & $\begin{array}{c}4 \\
(17.4)\end{array}$ & $\begin{array}{c}0 \\
(0)\end{array}$ & $\begin{array}{c}30 \\
(81.1)\end{array}$ & $\begin{array}{c}2 \\
(5.4)\end{array}$ & $\begin{array}{c}5 \\
(13.5)\end{array}$ & $\begin{array}{c}41 \\
(78.8)\end{array}$ & $\begin{array}{c}8 \\
(15.4)\end{array}$ & $\begin{array}{c}3 \\
(5.8)\end{array}$ & $\begin{array}{c}29 \\
(96.7)\end{array}$ & $\begin{array}{c}1 \\
(3.3)\end{array}$ & $\begin{array}{c}0 \\
(0)\end{array}$ & $\begin{array}{c}119 \\
(83.8)\end{array}$ & $\begin{array}{c}15 \\
(10.6)\end{array}$ & $\begin{array}{c}8 \\
(5.6)\end{array}$ \\
\hline 4. The disease may spread from bovine to sheep and goat & $\begin{array}{c}9 \\
(37.5) \\
\end{array}$ & $\begin{array}{c}7 \\
(29.2)\end{array}$ & $\begin{array}{c}8 \\
(33.3) \\
\end{array}$ & $\begin{array}{c}22 \\
(61.1)\end{array}$ & $\begin{array}{c}7 \\
(19.4) \\
\end{array}$ & $\begin{array}{c}7 \\
(19.4)\end{array}$ & $\begin{array}{c}32 \\
(61.5)\end{array}$ & $\begin{array}{c}10 \\
(19.2)\end{array}$ & $\begin{array}{c}10 \\
(19.2)\end{array}$ & $\begin{array}{c}23 \\
(82.1)\end{array}$ & $\begin{array}{c}0 \\
(0)\end{array}$ & $\begin{array}{c}5 \\
(17.9)\end{array}$ & $\begin{array}{c}86 \\
(61.4) \\
\end{array}$ & $\begin{array}{c}24 \\
(17.1)\end{array}$ & $\begin{array}{c}30 \\
(21.4)\end{array}$ \\
\hline 5. The disease may spread from sheep and goat to bovine & $\begin{array}{c}8 \\
(36.4) \\
\end{array}$ & $\begin{array}{c}7 \\
(31.8) \\
\end{array}$ & $\begin{array}{c}7 \\
(31.8) \\
\end{array}$ & $\begin{array}{c}21 \\
(58.3) \\
\end{array}$ & $\begin{array}{c}8 \\
(22.2) \\
\end{array}$ & $\begin{array}{c}7 \\
(19.4) \\
\end{array}$ & $\begin{array}{c}33 \\
(62.3) \\
\end{array}$ & $\begin{array}{c}7 \\
(13.2) \\
\end{array}$ & $\begin{array}{c}13 \\
(24.5) \\
\end{array}$ & $\begin{array}{c}20 \\
(69.0) \\
\end{array}$ & $\begin{array}{c}2 \\
(6.9) \\
\end{array}$ & $\begin{array}{c}7 \\
(24.1) \\
\end{array}$ & $\begin{array}{c}82 \\
(58.6) \\
\end{array}$ & $\begin{array}{c}24 \\
(17.1) \\
\end{array}$ & $\begin{array}{c}34 \\
(24.3) \\
\end{array}$ \\
\hline $\begin{array}{l}\text { 6. The GOI implemented control programme on brucellosis is fool } \\
\text { proof and will be successful }\end{array}$ & $\begin{array}{c}14 \\
(63.6)\end{array}$ & $\begin{array}{c}8 \\
(36.4)\end{array}$ & $\begin{array}{c}0 \\
(0)\end{array}$ & $\begin{array}{c}26 \\
(74.3)\end{array}$ & $\begin{array}{c}5 \\
5 \\
(14.3)\end{array}$ & $\begin{array}{c}4 \\
(11.4)\end{array}$ & $\begin{array}{c}26 \\
(54.2)\end{array}$ & $\begin{array}{c}15 \\
(31.3)\end{array}$ & $\begin{array}{c}7 \\
(14.6)\end{array}$ & $\begin{array}{c}6 \\
(20.0)\end{array}$ & $\begin{array}{c}23 \\
(76.7)\end{array}$ & $\begin{array}{c}1 \\
(3.3)\end{array}$ & $\begin{array}{c}72 \\
(53.3)\end{array}$ & $\begin{array}{c}51 \\
(37.8)\end{array}$ & $\begin{array}{c}12 \\
(8.9)\end{array}$ \\
\hline $\begin{array}{l}\text { 7. The test and slaughter policy will yield better results than } \\
\text { vaccination }\end{array}$ & $\begin{array}{c}10 \\
(40.0) \\
\end{array}$ & $\begin{array}{r}6 \\
(24.0) \\
\end{array}$ & $\begin{array}{c}9 \\
(36.0) \\
\end{array}$ & $\begin{array}{c}17 \\
(45.9) \\
\end{array}$ & $\begin{array}{c}9 \\
(24.3) \\
\end{array}$ & $\begin{array}{c}11 \\
(29.7) \\
\end{array}$ & $\begin{array}{c}18 \\
(35.3) \\
\end{array}$ & $\begin{array}{c}13 \\
(25.5) \\
\end{array}$ & $\begin{array}{c}20 \\
(39.2) \\
\end{array}$ & $\begin{array}{c}19 \\
(63.3) \\
\end{array}$ & $\begin{array}{c}7 \\
(23.3) \\
\end{array}$ & $\begin{array}{c}4 \\
(13.3) \\
\end{array}$ & $\begin{array}{c}64 \\
(44.8) \\
\end{array}$ & $\begin{array}{c}35 \\
(24.5) \\
\end{array}$ & $\begin{array}{c}44 \\
(30.8) \\
\end{array}$ \\
\hline $\begin{array}{l}\text { 8. Tagging will be helpful for tracking the disease spread in Indian } \\
\text { condition }\end{array}$ & $\begin{array}{c}17 \\
(68.0) \\
\end{array}$ & $\begin{array}{c}5 \\
(20.0) \\
\end{array}$ & $\begin{array}{c}3 \\
(12.0) \\
\end{array}$ & $\begin{array}{c}24 \\
(68.6) \\
\end{array}$ & $\begin{array}{c}6 \\
(17.1) \\
\end{array}$ & $\begin{array}{c}5 \\
(14.3) \\
\end{array}$ & $\begin{array}{c}40 \\
(78.4) \\
\end{array}$ & $\begin{array}{c}6 \\
(11.8) \\
\end{array}$ & $\begin{array}{c}5 \\
(9.8) \\
\end{array}$ & $\begin{array}{c}23 \\
(76.7) \\
\end{array}$ & $\begin{array}{c}5 \\
(16.7) \\
\end{array}$ & $\begin{array}{c}2 \\
(6.7) \\
\end{array}$ & $\begin{array}{c}104 \\
(73.8) \\
\end{array}$ & $\begin{array}{c}22 \\
(15.6) \\
\end{array}$ & $\begin{array}{c}15 \\
(10.6) \\
\end{array}$ \\
\hline $\begin{array}{l}\text { 9. Marginal, small and landless farmers have the capacity to pay for } \\
\text { vaccination if it is priced? }\end{array}$ & $\begin{array}{c}3 \\
(12.0)\end{array}$ & $\begin{array}{c}5 \\
(20.0)\end{array}$ & $\begin{array}{c}17 \\
(68.0)\end{array}$ & $\begin{array}{c}7 \\
(18.9)\end{array}$ & $\begin{array}{c}3 \\
(8.1)\end{array}$ & $\begin{array}{c}27 \\
(73.0)\end{array}$ & $\begin{array}{c}9 \\
(17.6)\end{array}$ & $\begin{array}{c}8 \\
(15.7)\end{array}$ & $\begin{array}{c}34 \\
(66.7)\end{array}$ & $\begin{array}{c}11 \\
(36.7)\end{array}$ & $\begin{array}{c}4 \\
(13.3)\end{array}$ & $\begin{array}{c}15 \\
(50.0)\end{array}$ & $\begin{array}{c}30 \\
(21.0)\end{array}$ & $\begin{array}{c}20 \\
(14.0)\end{array}$ & $\begin{array}{c}93 \\
(65.0)\end{array}$ \\
\hline $\begin{array}{l}\text { 10. Livestock insured farmers will be forthcoming than } \\
\text { farmers for vaccination programme }\end{array}$ & $\begin{array}{c}11 \\
(45.8)\end{array}$ & $\begin{array}{c}4 \\
(16.7)\end{array}$ & $\begin{array}{c}9 \\
(37.5)\end{array}$ & $\begin{array}{c}25 \\
(67.6)\end{array}$ & $\begin{array}{c}4 \\
(10.8)\end{array}$ & $\begin{array}{c}8 \\
(21.6)\end{array}$ & $\begin{array}{c}24 \\
(48.0)\end{array}$ & $\begin{array}{c}17 \\
(34.0)\end{array}$ & $\begin{array}{c}9 \\
(18.0)\end{array}$ & $\begin{array}{c}11 \\
(36.7)\end{array}$ & $\begin{array}{c}13 \\
(43.3)\end{array}$ & $\begin{array}{c}6 \\
(20)\end{array}$ & $\begin{array}{c}71 \\
(50.4)\end{array}$ & $\begin{array}{c}38 \\
(27.0)\end{array}$ & $\begin{array}{c}32 \\
(22.7)\end{array}$ \\
\hline
\end{tabular}

farmers for vaccination programme

\begin{tabular}{llllllllllllllll}
$(45.8)$ & $(16.7)$ & $(37.5)$ & $(67.6)$ & $(10.8)$ & $(21.6)$ & $(48.0)$ & $(34.0)$ & $(18.0)$ & $(36.7)$ & $(43.3)$ & $(20)$ & $(50.4)$ & $(27.0)$ & $(22.7)$ \\
\hline
\end{tabular}

Some of the respondents did not answered some of the attitude statements hence there is difference in number of respondents across the statements; A-agree, N-Neutral, DA-disagree

Table 5 Attitude Index on brucellosis in different surveyed states.

\begin{tabular}{|c|c|c|c|c|c|c|c|c|c|c|}
\hline \multirow{2}{*}{$\begin{array}{l}\text { Attitude } \\
\text { Index }\end{array}$} & \multicolumn{2}{|c|}{ Assam } & \multicolumn{2}{|c|}{ West Bengal } & \multicolumn{2}{|c|}{ Uttar Pradesh } & \multicolumn{2}{|c|}{ Punjab } & \multicolumn{2}{|c|}{ Pooled } \\
\hline & No. of respondents & Cumulative & No. of respondents & Cumulative & No. of respondents & Cumulative & No. of respondents & Cumulative & No. of respondents & Cumulative \\
\hline 6 to 19 & - & 0 & $1(2.6)$ & $1(2.6)$ & - & 0 & $1(3.3)$ & $1(3.2)$ & $2(1.4)$ & $2(1.4)$ \\
\hline 19 to 32 & - & 0 & - & $1(2.6)$ & $1(1.9)$ & $1(1.9)$ & - & $1(3.2)$ & $1(0.6)$ & $3(2.0)$ \\
\hline 32 to 45 & - & 0 & $1(2.6)$ & $2(5.1)$ & $1(1.9)$ & $2(3.8)$ & $1(3.3)$ & $2(3.3)$ & $3(2.0)$ & $6(4.0)$ \\
\hline 45 to 58 & $11(44.0)$ & $11(44.0)$ & $4(10.3)$ & $6(15.4)$ & $4(7.5)$ & $6(11.3)$ & $2(6.7)$ & $4(12.9)$ & $21(14.2)$ & $27(18.2)$ \\
\hline 58 to 71 & $12(48.0)$ & $23(92.0)$ & 19(48.7) & $25(64.1)$ & $32(60.4)$ & $38(71.7)$ & $15(50.0)$ & $19(61.3)$ & $78(52.6)$ & $105(70.8)$ \\
\hline 71 to 84 & $2(8.0)$ & $25(100.0)$ & $10(25.6)$ & $35(89.7)$ & $13(24.5)$ & $51(96.2)$ & $12(40.0)$ & $31(100.0)$ & $37(25.0)$ & 142(95.9) \\
\hline 84 to 97 & - & 0 & $4(10.3)$ & $39(100.0)$ & $2(3.8)$ & $53(100.0)$ & - & $31(100.0)$ & $6(4.1)$ & $148(100.0)$ \\
\hline Total & $25(100.0)$ & & $39(100.0)$ & & $53(100.0)$ & & $31(100.0)$ & & $148(100.0)$ & \\
\hline
\end{tabular}

Figures in parenthesis indicates percentage to the total

Journal of Experimental Biology and Agricultural Sciences

http://www.jebas.org 
Table 6 Results of practice levels by veterinarians to prevent the spread of brucellosis in the study states.

\begin{tabular}{|c|c|c|c|c|c|c|c|c|c|c|}
\hline & \multicolumn{2}{|c|}{ Assam } & \multicolumn{2}{|c|}{ West Bengal } & \multicolumn{2}{|c|}{ Uttar Pradesh } & \multicolumn{2}{|c|}{ Punjab } & \multicolumn{2}{|c|}{ Pooled } \\
\hline Practice statements & Practiced & $\begin{array}{c}\text { Not } \\
\text { practiced }\end{array}$ & Practiced & $\begin{array}{c}\text { Not } \\
\text { practiced }\end{array}$ & Practiced & $\begin{array}{c}\text { Not } \\
\text { practiced }\end{array}$ & Practiced & $\begin{array}{c}\text { Not } \\
\text { practiced }\end{array}$ & Practiced & Not practiced \\
\hline $\begin{array}{l}\text { 1. Obtain complete clinical history about the Brucella suspected } \\
\text { animal }\end{array}$ & $17(70.8)$ & $7(29.2)$ & $29(80.6)$ & $7(19.4)$ & $37(77.1)$ & $11(22.9)$ & $28(93.3)$ & $2(6.7)$ & $111(80.4)$ & $27(19.6)$ \\
\hline $\begin{array}{l}\text { 2. Gloves, goggles and mask should be used before attending } \\
\text { parturition }\end{array}$ & $21(84.0)$ & $4(16.0)$ & $18(52.9)$ & $16(47.1)$ & $33(68.8)$ & $15(31.3)$ & $22(75.9)$ & $7(24.1)$ & 94(69.1) & $42(30.9)$ \\
\hline $\begin{array}{l}\text { 3. Send clinical samples of suspected animals for confirmatory } \\
\text { diagnosis }\end{array}$ & $21(87.5)$ & $3(12.5)$ & $27(75.0)$ & $9(25.0)$ & 19(40.4) & $28(59.6)$ & $27(90.0)$ & $3(10.0)$ & $94(68.6)$ & $43(31.4)$ \\
\hline 4. Advice farmers about the proper disposal of infected materials & $23(95.8)$ & $1(4.2)$ & $31(86.1)$ & $5(13.9)$ & $42(89.4)$ & $5(10.6)$ & $28(96.6)$ & $1(3.4)$ & $124(91.2)$ & $12(8.8)$ \\
\hline $\begin{array}{l}\text { 5. Advice farmers to buy animals after assessing the history of } \\
\text { brucellosis symptoms }\end{array}$ & $19(82.6)$ & $4(17.4)$ & $21(61.8)$ & $13(38.2)$ & $38(80.9)$ & $9(19.1)$ & $20(69.0)$ & $9(31.0)$ & $98(73.7)$ & $35(26.3)$ \\
\hline $\begin{array}{l}\text { 6. Advice farmers not to use Brucella infected animal for natural } \\
\text { service }\end{array}$ & $21(87.5)$ & $3(12.5)$ & $31(86.1)$ & $5(13.9)$ & $43(91.5)$ & $4(8.5)$ & $28(96.6)$ & $1(3.4)$ & $123(90.4)$ & 13(9.6) \\
\hline
\end{tabular}

Table 7 Practice Index on brucellosis in different surveyed states.

\begin{tabular}{|c|c|c|c|c|c|c|c|c|c|c|}
\hline \multirow{2}{*}{$\begin{array}{l}\text { Practice } \\
\text { Index }\end{array}$} & \multicolumn{2}{|c|}{ Assam } & \multicolumn{2}{|c|}{ West Bengal } & \multicolumn{2}{|c|}{ Uttar Pradesh } & \multicolumn{2}{|c|}{ Punjab } & \multicolumn{2}{|c|}{ Pooled } \\
\hline & No. of respondents & Cumulative & No. of respondents & Cumulative & No. of respondents & Cumulative & No. of respondents & Cumulative & No. of respondents & Cumulative \\
\hline 16 to 30 & - & 0 & - & 0 & $1(2.2)$ & $1(2.2)$ & - & 0 & $1(0.8)$ & $1(0.8)$ \\
\hline 30 to 44 & $2(8.0)$ & $2(8.0)$ & - & 0 & $3(6.5)$ & $4(8.7)$ & - & 0 & $5(3.8)$ & $6(4.5)$ \\
\hline 44 to 58 & $2(8.0)$ & $4(16.0)$ & $1(3.2)$ & $1(3.2)$ & $4(8.7)$ & $8(17.4)$ & $2(6.7)$ & $2(6.7)$ & $9(6.8)$ & $15(11.4)$ \\
\hline 58 to 72 & $3(12.0)$ & $7(28.0)$ & $11(35.5)$ & $12(38.7)$ & $10(21.7)$ & $18(39.1)$ & $4(13.3)$ & $6(20.0)$ & $28(21.2)$ & $43(32.6)$ \\
\hline 72 to 86 & $8(32.0)$ & $15(60.0)$ & $6(19.4)$ & $18(58.1)$ & $13(28.3)$ & $31(67.4)$ & $10(33.3)$ & $16(53.3)$ & $37(28.0)$ & $80(60.6)$ \\
\hline 86 to 100 & $10(40.0)$ & $25(100.0)$ & $13(41.9)$ & $31(100.0)$ & $15(32.6)$ & $46(100.0)$ & $14(46.7)$ & $30(100.0)$ & $52(39.4)$ & $132(100.0)$ \\
\hline Total & $25(100.0)$ & & $31(100.0)$ & & $46(100)$ & & $30(100.0)$ & & $132(100.0)$ & \\
\hline
\end{tabular}

Figures in parenthesis indicates percentage to the total 
The questionnaire comprise basic demographic information viz., name, designation, working region, age, education qualification, years of service etc. besides various statements on brucellosis to assess the KAP level of veterinarians. The description and formation of questions to collect information on KAP parameters is presented in Table 1. The knowledge component of the questionnaire were divided into Brucella organism, clinical signs, source of infection in livestock, causes of brucellosis in humans, vaccination, Brucellosis CP and other aspects. The number of questions varied in different components/groups of the knowledge statement. The questions on KAP parameters comprised of both negative and positive statements. The negative statements were included as a part of the knowledge/attitude evaluation in order to differentiate the assessment clearly.

Based on the statement type (positive and negative) the scores were awarded. The positive statements were awarded larger score compared to negative statement. The two point assessment for knowledge (yes; no) and practice statements (practiced; not practiced), and three point Likert scale (agree; neutral and disagree) for attitude was considered. The scores for positive statements are yes (1) and no (0) and for negative statements yes (0) and no (1) to assess knowledge level. Similarly, for evaluating the practice/adoption level each statement was awarded one or zero based on practice/not practiced by the individual veterinarians. For attitude assessment, the scores for positive statements were (Agree-3; Neutral-2 and Disagree-1) and for negative statements the scores were (Agree-1; Neutral-2; Disagree-3). The information on different aspects of brucellosis was collected from the veterinarians with their oral consent to participate in the survey.

\subsection{Statistical analysis}

Indexes for knowledge, attitude and practice were calculated for the individual respondents and overall for the surveyed states instead of raw scores for easy comprehension. The Index was calculated based on Rahman, 2007.

a) Individual Knowledge Index (KI) was calculated by

$\mathrm{KI}=(\mathrm{AK} / \mathrm{MK}) * 100$

Where, $\mathrm{KI}=$ Knowledge Index, $\mathrm{AK}=$ Actual scoring of respondents for knowledge statements, $\mathrm{MK}=$ Maximum score for knowledge statements

b) Overall Knowledge Index was calculated by

$\mathrm{OKI}=\sum \mathrm{KI} / \mathrm{n}$

Where, $\sum \mathrm{KI}=$ Summation of individual Knowledge Index, $\mathrm{n}$ $=$ Total number of respondents, Similar formulae were used to calculate individual and overall Practice and Attitude index.

c) Sturges rule was employed for classifying the number of classes in knowledge, attitude and practice Index. d) Correlation (r) was used to determine the relationship between Index (KI or AI or PI) with various demographic parameters like age, number of years of service, education and also with prevalence level of brucellosis in their respective states.

e) The non-parametric Kruskal -Wallis Test was employed to compare the levels of knowledge, attitude and practice Index across the states surveyed

$\mathrm{KW}=\frac{12}{n(n+1)} \sum_{j=1}^{k} \frac{R^{2}}{n_{j}}-3(n+1)$

Where,

$\mathrm{KW}=$ Kruskal-Wallis value

$\mathrm{k}=$ number of groups

$\mathrm{NJ}=$ size of the $j$ th group

$\mathrm{RJ}=$ rank sum for the $j$ th group

$\mathrm{n}=$ total sample size

\section{Results}

\subsection{Socio-demographic characteristics of the respondents}

The age, education and experience details of the respondents in different surveyed states revealed that the overall median age and experience of the veterinarians was 44 years and 19 years, respectively.

\subsection{Knowledge}

Knowledge provides the basis for human behaviour and action. In the present study the knowledge of the veterinarians on brucellosis was assessed through set of statements on Brucella organism, clinical signs, and source of infection in livestock, causes of brucellosis in humans, vaccination, Brucellosis CP etc. The knowledge on various aspects across the states is summarised in Table 2. The state level results revealed that in Assam, $60 \%$ of the veterinarians had comprehensive knowledge (gave correct answers for all statements whereas it was less in other states (West Bengal, Uttar Pradesh, and Punjab) indicating differences in knowledge level on brucellosis in the surveyed states. The pooled results revealed that 32\% (4/4) of the respondents gave correct answers for all statements on Brucella organism per se whereas, $49 \%$ (3/4), $15 \%(2 / 4)$, and $4 \%(1 / 4)$ of the respondents, gave $75 \%, 50 \%$ and $25 \%$ correct answers, respectively. The knowledge on clinical signs of brucellosis was generally high across the states, whereas, the knowledge level on source of infection in livestock varied across the state (Punjab and West Bengal (75\%), Uttar Pradesh (57\%) and Assam (24\%)) implying the need for intervention to improve knowledge level of veterinarians. 
In all the study states less comprehensive knowledge (gave correct answers to all the questions) on causes/chances of brucellosis was observed. On vaccine and vaccination statements, around $68 \%$ and $66 \%$ of the veterinarians from West Bengal and Punjab, respectively were completely aware, whereas, it was only less than $30 \%$ in other states indicating variation in knowledge level on vaccine and vaccination details against brucellosis. In the study area less veterinarians had comprehensive knowledge on Brucellosis $\mathrm{CP}$ indicating the wide knowledge gap and necessity of training the veterinarians on different aspects on Brucellosis CP. The results on the knowledge level on susceptibility of breeds, age groups and hazard nature for the professionals revealed that $68 \%$ and $55 \%$ of the veterinarians from Uttar Pradesh and West Bengal respectively were fully aware on these aspects.

\subsection{Knowledge Index}

The Knowledge Index (KI) in different states is summarised in Table 3. In all the surveyed states except West Bengal maximum number of the respondents was in 74-85 KI. In West Bengal, the maximum number of respondents was in 85-96 range indicating the better knowledge level in this state compared to other surveyed states.

\subsection{Attitude}

Positive attitude combined with comprehensive knowledge especially among the field veterinarians will help to bolster the brucellosis control initiatives of the Government. The attitude results revealed that across the study states more than two-third $(67 \%)$ of the veterinarians agreed that brucellosis is a very important disease causing huge economic loss to any other disease in cattle (Table 4). There were wide differences in agreement across the states to the statement "brucellosis is the top most bacterial disease reported in the country" viz., Assam (30\%), West Bengal (32\%), Punjab (33\%) and Uttar Pradesh $(16 \%)$. In the study states, majority $(84 \%)$ of respondents believed that the chance of brucellosis spread in India's rural setting is fast compared to western countries. For the disease transmission statement from sheep and goat to bovines and vice versa, irrespective of the state, majority of the respondents believed that both way transmissions take place in livestock. Higher proportion of veterinarians (Assam (64\%), West Bengal (74\%), and Uttar Pradesh (54\%) except in Punjab state, opined that the Brucellosis CP implemented by Government of India will be successful in controlling the disease.

Though the test and slaughter policy for brucellosis disease control might yield better results than vaccination, majority of the respondents were divided on the issue. The majority (74\%) of the veterinarians in the study states exhibited positive attitude towards tagging the diseased animal and tracking the same to control the brucellosis spread. The opinions were varied across the study states among veterinarians for the statement "marginal, small and landless farmers have the capacity to pay for vaccination if priced? and "Livestock insured farmers will be forthcoming than non-insured farmers for vaccination programme" due to attitude differences (Table 4).

\subsection{Attitude Index}

The attitude index of the veterinarians revealed that majority $(60 \%)$ of the veterinarians in Uttar Pradesh had between 58 to 71 , whereas, it was 50\%, $49 \%$ and $48 \%$ respondents in Punjab, West Bengal and Assam, respectively. The pooled results revealed that majority of the respondent's attitude ranged from 58-71 (Table 5).

\subsection{Practice}

Besides the knowledge and attitude levels, the practice of the veterinarians, especially, when handling the suspected brucellosis case is an important in controlling the disease. Majority of the veterinarians in the surveyed states obtain complete clinical history of the animals suspected with brucellosis, but still 7 to $29 \%$ of the respondents are not collecting complete history (Table 6). The proportion of veterinarians using gloves, goggles and mask during parturition varied across the states viz., Assam (84\%), West Bengal (53\%), Uttar Pradesh (69\%) and Punjab (76\%). Sending clinical samples of the suspected animals for confirmatory diagnosis is not practiced by all the veterinarians. Advising farmers on the proper disposal of infected materials is practiced by majority of the respondents across the states (Assam (96\%), West Bengal (86\%), Uttar Pradesh (89\%) and Punjab (97\%)). Similar results were obtained for the practice statements 'Advice farmers to buy animals after assessing the history of brucellosis symptoms' and 'Advice farmers not to use brucellosis infected animals for natural service'.

\subsection{Practice Index}

The practice index is very important since any deviation from the set procedure in treating the animals will widen the scope of infection and spread of brucellosis. In all the surveyed states the practice index levels among the veterinarians was less (Table 7). The cumulative practice levels of respondents revealed that in Uttar Pradesh two-third of the veterinarians had less than 86 index level, whereas in Assam, West Bengal and Punjab the proportion of respondents were $60 \%, 58 \%$ and $53 \%$, respectively. 
3.8 Correlation of knowledge level vs demographic variables and prevalence

No significant correlation was observed between the personal attributes and knowledge level of the veterinarians in all the states except Punjab (Table 8). In Assam, Uttar Pradesh and Punjab significant positive correlation $(\mathrm{p}<0.01)$ exists between brucellosis prevalence level in the state and the knowledge level of the veterinarians. The attitude and practice level of the respondents is not significantly correlated with the personal attributes in all the studied states of India ( $p>0.01)$, whereas, it is significantly correlated with prevalence level of Assam, Uttar Pradesh and Punjab $(\mathrm{p}<0.01)$. The results of nonparametric Kruskal-Wallis test revealed that there exists significant difference in knowledge and attitude level across the states $(p<0.01)$, whereas, no significant difference in practice index across the states $(\mathrm{p}>0.01)$.

\section{Discussions}

Literature revealed that there is no information available on the knowledge, attitude and practice level of veterinarians on brucellosis in India for an important zoonotic disease like brucellosis, which has inherent difficulties in treatment and prevention due to various factors. In a federal country like India, the state administration play an important role in implementing various livestock and human resource development programs and hence, the state level analysis on KAP were carried out however, generalizing the results of this study should be approached cautiously due to region specific, small sample size and self reporting by the veterinarians. There were wide differences in knowledge about the type of organism, its nature of residence within host, its complexity in infecting various hosts. The differences might be due to various levels of knowledge updating, especially after joining field veterinary service, though it is needed among the risk groups to avoid zoonotic infections.

The knowledge on clinical signs is very important to suspect a case for brucellosis at field level. Around 60\%, 80\%, 84\% and $76 \%$ of respondents from Assam, West Bengal, Uttar Pradesh and Punjab were aware of all the major clinical signs, but comprehensive knowledge should be imparted to field veterinarians. The results are in line with Adesiji et al. (2005), who reported that among 540 respondents who include butchers, herdsmen, meat vendors, milkers and veterinarians, only $19.6 \%$ are aware of the etiology, mode of transmission and prevention of brucellosis.

He also highlighted that there was a high level of awareness among the veterinarians than other groups studied. Same was observed in findings of Bilal et al. (1991) that out of 337 subjects examined for knowledge about methods and means of transmission of brucellosis, 309 (92\%) were ignorant. There were wide differences in the knowledge on sources of Brucella infection in cattle and humans. Since Brucella is a zoonotic disease, the complete knowledge on infection in cattle and humans is essential to prevent its infection and spread to 
livestock, veterinarians and their peer groups. It also helps the veterinarians to disseminate appropriate information to the farmers/farm animal handler to protect this deadly disease spread. The knowledge levels on vaccine and vaccination is very low in many states and it may be due to the fact that vaccine and vaccination policies in different countries are varying (S19 vs RB51 strain; Calf hood vs adult vaccination).

Brucellosis CP has been implemented in India since 2010, however, the knowledge level of the field veterinarians on Brucellosis CP was very low. Hence, detailed plan of Brucellosis $\mathrm{CP}$ as well as uniform guidelines on vaccination and surveillance has to be made available to the field veterinarians through the respective state governments for its effective implementation. Though the maximum numbers of respondents across the states were in the range of 74-85 knowledge index, there were few respondents with very low Knowledge Index. It implies the necessity of appropriate training on the theoretical and practical aspects on brucellosis disease to upgrade the knowledge levels of field veterinarians (Mazloomy et al., 2008). Hence, training might be the best option to achieve comprehensive knowledge on brucellosis among the veterinarians.

Across the states majority of the veterinarians agreed that brucellosis is a very important disease causing huge economic loss to any other disease in cattle. Though brucellosis may not inflict more direct losses like infectious diseases, but, its zoonotic nature will have wider social and economic ramifications and hence, majority respondents might believed that brucellosis causes huge economic loss. It is also evident from McDermott et al., (2013), that brucellosis is consistently ranked among the most economically important zoonoses globally. It is a 'multiple burdens' disease with economic impacts attributable to human, livestock and wildlife disease. Though Haemorrhagic Septicaemia (HS) disease in livestock is the top most bacterial disease reported in India (Benkirane et al., 2002), many veterinarians perceived that brucellosis as the top most bacterial disease reported implying lack of awareness about the macro disease scenario in the country. Majority of the respondents attitude towards the spread of brucellosis in India's rural setting is fast compared to developed countries and it might be due to lack of facilities for 'screening of all the animals for brucellosis' in India's rural setting and also due to policy ban on slaughter of cattle in majority of the states.

Also due to trade, rapid movement of livestock and preponderance of natural bull service in rural India (Lindahl et al., 2015) are the other compelling reasons to believe faster spread of brucellosis in India's rural setting. The majority of the respondents have positive attitude towards the tagging the diseased animal since it assists in tracking the animal. Majority of the respondents disagreed to the statement of pricing of the vaccine for marginal, small and landless farmers indicating the strong need for free public intervention to control brucellosis. Across the states the respondent's overall Attitude Index (5871) was less than Knowledge Index (74-85) indicating more scope for changing the attitude through appropriate intervention.

Before handling the Brucella suspected animal, obtaining the complete history is important, but, in the surveyed states few veterinarians (7 to 29\%) are not following this practice. Similarly, during the parturition majority of the veterinarians are not using all the major gadgets like gloves, goggles and mask. Majority of the respondents opined that they use only gloves mainly due to non-availability of other protective gears from the department. The results are on par with Hannah et al. (2011), who reported that only $21 \%$ of the total respondents used protective gears while dealing with cows having an abortion or with aborted materials. This shows there exists high risk of brucellosis spread among field veterinarians. Adesiji et al. (2005) also reported that presence of open wounds while handling diseased animal caused brucellosis in three veterinarians among 10 veterinarians surveyed.

Hence, there is a necessity to spread awareness among the veterinarians on the repercussions of non-using of protective gears besides ensuring the regular supply of these gears by the Government. Sending clinical samples of the suspected animals for confirmatory diagnosis is not practiced by all the veterinarians due to practical difficulties in field conditions. It may also accentuate the disease spread to many animals and humans in the long-run.

On the contrary, even if diagnosed, lack of national level compensation policy and ban on cow slaughter in various states of India may lead to distress sale and un-intended spread. Advising farmers on different facets of the brucellosis disease is not followed by all the veterinarians and hence, all the veterinarians should be stressed to educate farmers on the importance of proper disposal of infected materials; purchasing of the animals after assessing the history of brucellosis and advocating farmers to refrain from natural service from the brucellosis infected bulls in order to control the brucellosis spread. In all the surveyed states the practice index levels was low implying majority of the respondents do not follow the standard practices. Hence, there is scope for improvement on the part of Government as well as veterinary professionals for controlling brucellosis infection and spread in livestock and humans in India.

The KAP level is not significantly correlated with the personal attributes of the respondents in majority of the study states. In Punjab, positive correlation was observed for knowledge level and demographic variables (age, education and experience levels) of the veterinarians and brucellosis prevalence level. There exists significant difference within knowledge level and attitude level across the states and no significant difference within practice index across the states. The significant difference across states might be due to differences in institutional and extension activities taken up by the states to upgrade the KAP levels of veterinarians. 


\section{Conclusions}

The comprehensive knowledge, attitude and set of practices in handling Brucella infected cases are not observed among the veterinarians. Hence, it warrants urgent intervention to sensitize the veterinarians through appropriate training and experiential learning techniques to upgrade the knowledge level and to build positive attitude. The field veterinarians need to be instructed to follow the set practices during the treatment of animals in order to avoid infection of brucellosis to themselves, peers and the society at large. The government investment is very much essential especially in providing the basic tools like gloves, goggles and mask to protect veterinarian and their staff from Brucella infection. The uniform guidelines for vaccination, surveillance, mechanism to handle diseased animals should also be disseminated to the field veterinarians appropriately to mitigate brucellosis infection and for the success of Brucellosis CP. Collective efforts in 'One Health' approach to improve knowledge, attitude and practice of veterinarians supported by appropriate government intervention will help to prevent the brucellosis infection and its spread among veterinarians, livestock farmers and related risk groups, thus making an initiative for one world one health approach.

\section{Acknowledgements}

Authors would like to thank Indian Council of Agricultural Research (ICAR) for providing constant support and encouragement for pursuing research and also thank the veterinarians from different states of India for participating in the survey; also the authors wish to thank DAHD\&F for providing grants-in-aid under the Livestock Health and Disease Control (LH and DC) to ICAR-NIVEDI to coordinate the surveillance and vaccination under Brucellosis control program and state Animal Husbandry Department for making this study feasible.

\section{Conflict of interest}

Authors would hereby like to declare that there is no conflict of interests that could possibly arise.

\section{References}

Adesiji YO, Adesiji GB, Fagbami AH (2005) Brucellosis: Knowledge, Attitude and Practices among occupationally exposed individuals in Osun state. Science focus Journal 10: $38-41$.

Bartlett JE, Kotrlik JW, Higgins CC (2001) Organizational Research: Determining Appropriate Sample Size in Survey Research. Information Technology, Learning, and Performance Journal 19: 43-50.

Benkirane A, De Alwis MCL (2002) Haemorrhagic septicaemia, its significance, prevention and control in Asia. VeterinarniMedicina-Czech 47: 234-240.
Bilal NE, Jamjoom GA, Bobo RA, Aly OF, el-Nashar NM (1991) A study of the knowledge, attitudes and practice (KAP) of a Saudi Arabian Community towards the problem of brucellosis. Journal of Egyptian Public Health Association 66: 227-238.

Department of Animal Husbandry, Dairying \& Fisheries DAHD\&F (2012) Basic Animal Husbandry and Fisheries Statistics.Government of India.Available at: http://dahd.nic.in/dahd/WriteReadData/Livestock.pdf.Accessed February 232015.

Gull ST, Khan A (2007) Epidemiology and Epizootiology of Brucellosis. A reviewPakistan Veterinary Journal 27: 145-151.

Hajia M, Rahbar M, Kermit F (2009) Epidemiological, clinical, diagnostic and treatment aspects of hospitalized Brucellosis patients in Hamadan. Annals of Tropical Medicine and Public Health 2: 42-45.

Hannah RH, Mahmud ME, Yemen MH, Weal FE, Ahmed AT, Javier G (2011) Brucella spp infection in large ruminants in an endemic area of Egypt: cross-sectional study investigating seroprevalence, risk factors and livestock owner's Knowledge, Attitudes and Practices (KAPs). BMC Public health 11: 341.

Kapoor PK, Sharma SN, Rao KL (1985) Seroprevalence of brucellosis in goats and human beings in Bikaner (Rajasthan). Indian Journal of Comparitive Microbiology Immunology and Infectious Diseases 6: 96-101.

Lindahl E, Sattorov N, Boqvist A, Magnusson U (2015) A study of Knowledge, Attitude and Practices relating to Brucellosis among small scale dairy farmers in an urban and peri-urban area of Tajikistan. PLoS ONE 10(2): e0117318.http://dx.doi.org/10.1371/journal.pone.0117318

Londhe SP, Bannalikar AS, Dighe VD (2011)Serodetection of bovine brucellosis by RBPT and AB-ELISA.Animal Science Reporter5: 69-73.

MazloomyMahmoodabad SS, Barkhordari A, Nabizadeh M and Ayatollahi J (2008) The effect of health education on Knowledge, Attitude and Practice (KAP) of high school students towards Brucellosis in Yazd. World Applied Sciences Journal 5:522-524.

McDermott J, Grace D, Zinsstag J (2013) Economics of brucellosis impact and control in low-income countries.Revue scientifique et technique32: 249-261.

Mousa AR, Muhtaseb SA, Almudallal DS, Khodeir SM, Marafie AA (1987) Osteoarticular complications of brucellosis: a study of 169 cases. Reviews of Infectious Diseases 9: 531-543.

Panjarathinam R, Jhala CI (1986) Brucellosis in Gujarat state Indian Journal of Pathology and Microbiology29: 53-60. 
Radostits, Gay OMC, Blood DC, Hinchcliff KW (2000) Veterinary medicine: A textbook of the diseases of cattle, horses, sheep, pigs and goats. London, United Kingdom ELBS Bailliere Tindal: 870-871.

Rahman S (2007) Adoption of improved technologies by the pig farmers of Aizawl district. http://www.lrrd.org/lrrd19/1/rahm19005. Accessed April 17 2015.

Rajkhowa S, Rahman H, Rajkhowa C, Bujarbaruah KM (2005) Seroprevalence of brucellosis in mithuns (Bos frontalis) in India. Preventive Veterinary Medicine 69: 145-51. http://doi:10.1016/j.prevetmed.2005.02.005.

Ramesh VJ, Rajesh R, Karthik K and Ramesh S (2013) Seroprevalence studies of bovine brucellosis using indirect enzyme-linked immunosorbent assay (i-ELISA) at organized and unorganized farms in three different states of India. Veterinary World 6: 550-553.doi:10.5455/vetworld.2013.550553
Refai M (2002) Incidence and control of brucellosis is the near east region. Veterinary Microbiology. 90: 81-110.

Renukaradhya GJ, Isloor S, Rajasekhar M (2002) Epidemiology, zoonotic aspects, vaccination and control eradication of brucellosis in India. Veterinary Microbiology 90: 183-195. http://dx.doi.org/10.1016/S0378-1135(02)002535

Shome R, Nagarathna S, Prashant G, Nagalingam M, Padmashree K, Triveni K, Shome BR, Gupta VK, Rahman H (2014) Sexual Transmission of Human Brucellosis: Case Studies International Journal of Health Sciences and Research4: 61-67.

Skalsky K, Yahav D, Bishara J, Pitlik S, Leibovici L, Paul M (2008) Treatment of human brucellosis systematic review and meta-analysis of randomised controlled trials. BMJ9336: 701704. DOI: 10.1136/bmj.39497.500903.25. 